\title{
On the Solution of KdV-Like Equations by the Optimal Perturbation Iteration Technique
}

\author{
Necdet Bildik* \\ Manisa Celal Bayar University, Faculty of Arts \& Science, Department of Mathematics, Muradiye Campus, \\ 45140, Manisa, Turkey. \\ * Corresponding author. Tel.: +(90)0236 201 3203; email: necdet.bildik@cbu.edu.tr \\ Manuscript submitted March 10, 2018; accepted October 3, 2018. \\ doi: 10.17706/ijapm.2019.9.1.29-37
}

\begin{abstract}
In this study, optimal perturbation iteration method is implemented to solve Korteweg de Vries $(\mathrm{KdV})$-like equation to obtain semi analytical solutions. We examine two illustrations to analyze the new optimal perturbation iteration method. This work displays that optimal perturbation iteration technique converges fast to the exact solutions of the differential equations at lower order of approximations.
\end{abstract}

Key words: Optimal perturbation iteration method, KdV equation.

\section{Introduction}

Over the last century, much attention has been given to partial differential equations due to their usage to describe complex phenomena in various fields of science and engineering. Various methods have been constructed to get the more approximate solutions of linear and nonlinear partial differential equations. When the exact solutions do not exist, those approximate solutions provide important information about physical meanings behind the considered partial differential equations. Therefore numerous techniques have been devised to obtain the exact and approximate solutions of both ordinary and nonlinear partial differential equations [1]-[11].

The Korteweg-de Vries equation is used to model the waves on shallow water surfaces. It is especially noteworthy as a prototype example of a fully solvable model, that is, a non-linear partial differential equation whose solutions can be exactly defined. Over one hundred and fifty years ago, while conducting experiments to determine the most practical design for canal boats, a young Scottish engineer named John Russell (1808-1882) made a remarkable scientific revelation. A nice story about the history and the underlying physical properties of the Korteweg-de Vries equation can be found at an Internet page of the Herriot-Watt University in Edinburgh [12].

In this research, we take homogenous linear Korteweg-de Vries like equations as

$$
u_{t}+u_{x}+u_{x x x}=0, u(x, 0)=f(x), \quad 0 \leq x \leq 1, \quad t>0
$$

where $u=u(x, t)$ is the unknown function. Firstly, optimal perturbation iteration algorithm is constructed for the homogenous linear Korteweg-de Vries like equations. After that, approximate solutions are obtained by using these algorithms. Some figures will also be sketched to display the accuracy and effectiveness of the new technique. 


\section{OPIM for the Korteweg-de Vries Equation}

Optimal perturbation iteration method has been recently devised to deal with the nonlinear problems. It has been widely used to solve the ordinary and partial differential equations [13]-[19]. We now create an algorithm for solving homogenous linear Korteweg-de Vries like equations as follows:

a) The Eq. (1) can be taken as:

$$
F\left(u_{x x x}, u_{x}, u_{t}, \varepsilon\right)=0
$$

where $\varepsilon$ is the perturbation parameter can be put into the Eq. 2 as:

$$
F=\varepsilon\left(u_{x}+u_{x x x}\right)+u_{t}=0
$$

$F$ can be decomposed as:

$$
F=S+R
$$

to make computations easier. Here $S$ is the simpler part, which can be easily conducted and $R$ is the remaining part which is dominant for OPIM algorithms. For the equation (2), one can choose

$$
R\left(u_{x x x}, u_{x}, \varepsilon\right)=\varepsilon u_{x}+\varepsilon u_{x x x}
$$

and

$$
S\left(u_{t}\right)=u_{t}
$$

respectively.

b) The approximate solution with one correction term in the perturbation expansion can be taken as

$$
u_{n+1}=u_{n}+\varepsilon\left(u_{c}\right)_{n}
$$

where $\left(u_{c}\right)_{n}$ is the $n$th correction term. We then replace equation (6) into (3) to get an algorithm for OPIM. After substituting, we expand it in a series at $\varepsilon=0$ with first derivative as

$$
F+F_{u_{t}}\left(\left(u_{c}\right)_{n}\right)_{t} \varepsilon+F_{u_{x x}}\left(\left(u_{c}\right)_{n}\right)_{x x x} \varepsilon+F_{u_{x}}\left(\left(u_{c}\right)_{n}\right)_{x} \varepsilon+F_{\varepsilon} \varepsilon=0
$$

or equivalently

$$
R+R_{u_{t}}\left(\left(u_{c}\right)_{n}\right)_{t} \varepsilon+R_{u_{x x x}}\left(\left(u_{c}\right)_{n}\right)_{x x x} \varepsilon+R_{u_{x}}\left(\left(u_{c}\right)_{n}\right)_{x} \varepsilon+R_{\varepsilon} \varepsilon=-S
$$

Here, we use the following notations 


$$
R_{\varepsilon}=\frac{\partial R}{\partial \varepsilon}, R_{u_{t}}=\frac{\partial R}{\partial u_{t}}, R_{u_{x}}=\frac{\partial R}{\partial u_{x}}, R_{u_{x x x}}=\frac{\partial R}{\partial u_{x x x}}
$$

By performing the necessary calculations for the Eq. (3), we get

$$
\left(\left(u_{c}\right)_{n}\right)_{t}=-\left(u_{n}\right)_{x x x}-\left(u_{n}\right)_{x}-\left(u_{n}\right)_{t} .
$$

The above expression is the optimal perturbation iteration algorithm (OPIA) for the KdV-like equation (1). In order to start the OPIA iterations, $u_{0}$, an initial function is chosen appropriately according to the prescribed initial conditions.

c) The first correction term $u_{1}$ can be computed from the algorithm (10). In order to increase the effectiveness of technique and to get better results, we optimize the solutions by using the equation

$$
u_{n+1}=u_{n}+P_{n}\left(u_{c}\right)_{n}
$$

Here, we call $P_{0}, P_{1}, P_{2}, \ldots$ as convergence control parameters. These constants allow us to adjust the convergence. For $n=0,1, \ldots$, we have

$$
\begin{aligned}
& u_{1}=u\left(x, t ; P_{0}\right)=u_{0}+P_{0}\left(u_{c}\right)_{0} \\
& u_{2}\left(x, t ; P_{0}, P_{1}\right)=u_{1}+P_{1}\left(u_{c}\right)_{1} \\
& \quad \vdots \\
& u_{m}\left(x, t ; P_{0}, \ldots, P_{m-1}\right)=u_{m-1}+P_{m-1}\left(u_{c}\right)_{m-1}
\end{aligned}
$$

d) When $u_{m}$ is substituted into the equation (1), the overall problem will become:

$$
\operatorname{Re}\left(x, t ; P_{0}, \ldots, P_{m-1}\right)=F\left(\left(u_{m}\right)_{x x},\left(u_{m}\right)_{t t},\left(u_{m}\right)_{t}, u_{m}\right)
$$

or likewise

$$
\operatorname{Re}\left(x, t ; P_{0}, \ldots, P_{m-1}\right)=R\left(u_{m}\left(x, t ; P_{0}, \ldots, P_{m-1}\right)\right)+S\left(u_{m}\left(x, t ; P_{0}, \ldots, P_{m-1}\right)\right) .
$$

Apparently, when $\operatorname{Re}\left(x, t ; P_{0}, \ldots, P_{m-1}\right)=0$ then it means that $u_{m}\left(x, t ; P_{0}, \ldots, P_{m-1}\right)$ is the exact solution. However, such a situation will not usually occur for nonlinear equations, but one can minimize the functional as:

$$
J\left(P_{0}, \ldots, P_{m-1}\right)=\int_{0}^{T} \int_{a}^{b} R e^{2}\left(x, t ; P_{0}, \ldots, P_{m-1}\right) d x d t
$$

where $a, b$ and $T$ are picked on the domain of the problem. Finally, the convergence parameters 
$P_{0}, P_{1}, \ldots$ can be optimally reached by imposing the following equality

$$
\frac{\partial J}{\partial P_{0}}=\frac{\partial J}{\partial P_{1}}=\ldots=\frac{\partial J}{\partial P_{m-1}}=0
$$

The approximate solution of order $m$ can be obtained after putting $P_{0}, P_{1}, \ldots$ into the last one of the equations (12). For more detailed information about OPIM, please see [13-19].

\section{Illustrations}

To verify the validity and the potential of OPIM, we solve two examples about KdV like equations. The obtained results are also compared with the exact solutions to test the effectiveness of the recommended technique.

Example 1) Consider the following homogeneous linear KdV like equation [20],

$$
u_{x x x}+u_{x}+u_{t}=0
$$

with the initial conditions

$$
u(x, 0)=\sinh x \quad(0<x \leq 1, t \geq 0) .
$$

Firstly, a starting function can be taken as

$$
u_{0}=\sinh x \text {. }
$$

Then, by the help of equations (10) and (19) with initial conditions, first correction term can be calculated as:

$$
\left(u_{c}\right)_{0}=-2 t \cosh x
$$

Thus, the first order OPIM solution is found as follows:

$$
u_{1}=\sinh x-2 P_{0} t \cosh x .
$$

Using the same algorithm to find the other individual terms of the solutions, we get

$$
\begin{aligned}
u_{2}=\sinh [x]-2 t \cosh [x] P_{0}-\left(2 t \cosh [x]-2 t \cosh [x] P_{0}-2 t^{2} \sinh [x] P_{0}\right) P_{1} \\
u_{3}=\sinh [x]-2 t \cosh [x] P_{0}-\left(2 t \cosh [x]-2 t \cosh [x] P_{0}-2 t^{2} \sinh [x] P_{0}\right) P_{1}- \\
\left(\begin{array}{l}
2 t \cosh [x]-2 t \cosh [x] P_{0}-2 t^{2} \sinh [x] P_{0}-2 t \cosh [x] P_{1}-2 t^{2} \sinh [x] P_{1}+ \\
2 t \cosh [x] P_{0} P_{1}+\frac{4}{3} t^{3} \cosh [x] P_{0} P_{1}+4 t^{2} \sinh [x] P_{0} P_{1}
\end{array}\right) P_{2}
\end{aligned}
$$




$$
\begin{aligned}
& u_{4}=\sinh [x]-2 t \cosh [x] P_{0}-\left(2 t \cosh [x]-2 t \cosh [x] P_{0}-2 t^{2} \sinh [x] P_{0}\right) P_{1}- \\
& \left(\begin{array}{l}
2 t \cosh [x]-2 t \cosh [x] P_{0}-2 t^{2} \sinh [x] P_{0}-2 t \cosh [x] P_{1} \\
-2 t^{2} \sinh [x] P_{1}+2 t \cosh [x] P_{0} P_{1}+\frac{4}{3} t^{3} \cosh [x] P_{0} P_{1}+4 t^{2} \sinh [x] P_{0} P_{1}
\end{array}\right) P_{2}- \\
& \left(\begin{array}{l}
2 t \cosh [x]-2 t \cosh [x] P_{0}-2 t^{2} \sinh [x] P_{0}-2 t \cosh [x] P_{1}-2 t^{2} \sinh [x] P_{1}+ \\
2 t \cosh [x] P_{0} P_{1}+\frac{4}{3} t^{3} \cosh [x] P_{0} P_{1}+4 t^{2} \sinh [x] P_{0} P_{1}-2 t \cosh [x] P_{2}-2 t^{2} \sinh [x] P_{2}+ \\
2 t \cosh [x] P_{0} P_{2}+\frac{4}{3} t^{3} \cosh [x] P_{0} P_{2}+4 t^{2} \sinh [x] P_{0} P_{2}+2 t \cosh [x] P_{1} P_{2}+ \\
\frac{4}{3} t^{3} \cosh [x] P_{1} P_{2}+4 t^{2} \sinh [x] P_{1} P_{2}-2 t \cosh [x] P_{0} P_{1} P_{2}-4 t^{3} \cosh [x] P_{0} P_{1} P_{2} \\
-6 t^{2} \sinh [x] P_{0} P_{1} P_{2}-\frac{2}{3} t^{4} \sinh [x] P_{0} P_{1} P_{2}
\end{array}\right.
\end{aligned}
$$

and so on. In order to obtain the unknown parameters, we need to calculate the residuals for each order of approximate solutions. For instance, the following residual is constructed for the third order approximation as:

$$
\begin{aligned}
& \operatorname{Re}\left(x, t ; P_{0}, P_{1}, P_{2}\right)=\left(u_{3}\right)_{x x x}+\left(u_{3}\right)_{x}+\left(u_{3}\right)_{t}= \\
& 2\left(\cosh [x]-(\cosh [x]+2 t \sinh [x]) P_{2}+P_{1}\left(\begin{array}{l}
-\cosh [x]-2 t \sinh [x]+ \\
\left(\cosh [x]+2 t^{2} \cosh [x]+4 t \sinh [x]\right) P_{2}
\end{array}\right)\right) \\
& \left.\left.-\frac{2}{3} P_{0}\left(\begin{array}{l}
3\left(\cosh [x]+2 t \sinh [x]-\left(\cosh [x]+2 t^{2} \cosh [x]+4 t \sinh [x]\right) P_{2}\right)+ \\
P_{1}\left(-3\left(\cosh [x]+2 t^{2} \cosh [x]+4 t \sinh [x]\right)+\left(\begin{array}{l}
3\left(1+6 t^{2}\right) \cosh [x]+ \\
2 t\left(9+2 t^{2}\right) \sinh [x]
\end{array}\right)\right.
\end{array}\right) P_{2}\right)\right)
\end{aligned}
$$

For $a=0, b=1$ and $T=1$ the Eq. (15) becomes

$$
J\left(P_{0}, P_{1}, P_{2}\right)=\int_{0}^{1} \int_{0}^{1} \operatorname{Re}^{2}\left(x, t ; P_{0}, P_{1}, P_{2}\right) d x d t
$$

After computing the above integral, we need to solve the following equality

$$
\frac{\partial J}{\partial P_{0}}=\frac{\partial J}{\partial P_{1}}=\frac{\partial J}{\partial P_{2}}=\ldots=0
$$

to get the convergence control parameters. Upon substituting these parameters into the equation (23) and (24) respectively, we obtain the third and fourth order approximate OPIM solutions as 


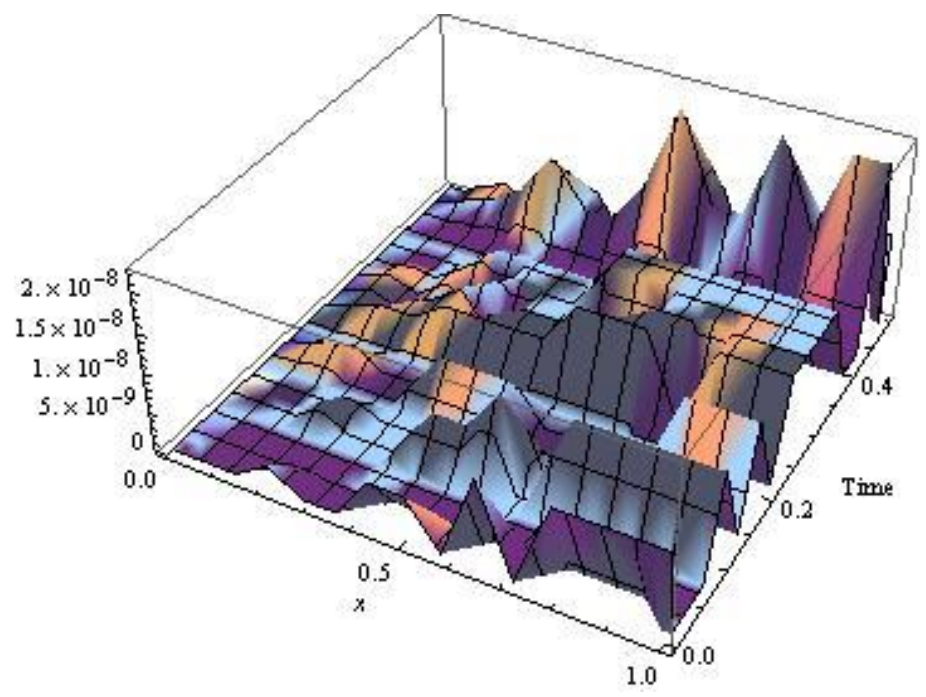

Fig. 1. Absolute errors for seventh order OPIM solution for example 1.

$u_{3}=-1.795987 t \cosh [x]+\sinh [x]-0.756153\left(0.2040129 t \cosh [x]-1.795987 t^{2} \sinh [x]\right)$

$-0.90112\left(0.049785 t \cosh [x]+0.90536 t^{3} \cosh [x]-0.59221 t^{2} \sinh [x]\right)$

$u_{4}=-1.97002205 t \cosh [x]+\sinh [x]-0.9805536\left(0.029977 t \cosh [x]-1.97002 t^{2} \sinh [x]\right)-$

$0.999020\left(0.000582 t \cosh [x]+1.2878081 t^{3} \cosh [x]-0.06770478 t^{2} \sinh [x]\right)-$

$\left(\begin{array}{l}5.7123337 \times 10^{-7} t \cosh [x]+0.0463541 t^{3} \cosh [x]- \\ 0.00064873 t^{2} \sinh [x]-0.6432731 t^{4} \sinh [x]\end{array}\right) P_{3}$

By continuing the computations, more approximate solutions can also be obtained using the algorithms. Fig. 1 displays the absolute errors for the 7th order OPIM solutions.

Example 2) Let us now consider the following homogeneous linear KdV like equation [20],

$$
u_{x x x}+u_{x}+u_{t}=0,(0<x \leq 1, t \geq 0)
$$

with the initial conditions $u(x, 0)=e^{-x}$.

The initial condition is the best candidate for being a test function, so we take

$$
u_{0}=e^{-x}
$$

With the aid of the equations (10), (12) and (30) we can start to construct the algorithms. Advancing as in first problem, we can find the following approximate OPIM results:

$$
\begin{gathered}
u_{1}=\mathrm{e}^{-x}+2 \mathrm{e}^{-x} t P_{0} \\
u_{2}=\mathrm{e}^{-x}+2 \mathrm{e}^{-x} t P_{0}-\left(-2 \mathrm{e}^{-x} t+2 \mathrm{e}^{-x} t P_{0}-2 \mathrm{e}^{-x} t^{2} P_{0}\right) P_{1}
\end{gathered}
$$




$$
\begin{aligned}
& u_{3}=\mathrm{e}^{-x}+2 \mathrm{e}^{-x} t P_{0}-\left(-2 \mathrm{e}^{-x} t+2 \mathrm{e}^{-x} t P_{0}-2 \mathrm{e}^{-x} t^{2} P_{0}\right) P_{1}- \\
& \left(-2 \mathrm{e}^{-x} t+2 \mathrm{e}^{-x} t P_{0}-2 \mathrm{e}^{-x} t^{2} P_{0}+2 \mathrm{e}^{-x} t P_{1}-2 \mathrm{e}^{-x} t^{2} P_{1}-2 \mathrm{e}^{-x} t P_{0} P_{1}+4 \mathrm{e}^{-x} t^{2} P_{0} P_{1}-\frac{4}{3} \mathrm{e}^{-x} t^{3} P_{0} P_{1}\right) P_{2}
\end{aligned}
$$

and so on. After finding these approximations, corresponding residual can be computed as:

$$
\operatorname{Re}\left(x, t ; P_{0}, P_{1}, P_{2}\right)=-\frac{2}{3} \mathrm{e}^{-x}\left(\begin{array}{l}
3\left(1+(-1+2 t) P_{2}+P_{1}\left(-1+2 t+\left(1-4 t+2 t^{2}\right) P_{2}\right)\right)+ \\
P_{0}\left(3\left(-1+2 t+\left(1-4 t+2 t^{2}\right) P_{2}\right)+P_{1}\left(\begin{array}{l}
3-12 t+6 t^{2}+ \\
\left(-3+18 t-18 t^{2}+4 t^{3}\right) P_{2}
\end{array}\right)\right)
\end{array}\right)
$$

for the third order OPIM approximate solution. Solving the equality (26) and (27) and substituting the appropriate constants into the suitable solutions, we can get the following approximate results:

$$
\begin{gathered}
u_{3}=\mathrm{e}^{-x}+1.995987 \mathrm{e}^{-x} t-0.956153\left(-0.004012 \mathrm{e}^{-x} t-1.995987 \mathrm{e}^{-x} t^{2}\right) \\
-0.991123\left(-0.000175 \mathrm{e}^{-x} t-0.0913541 \mathrm{e}^{-x} t^{2}-1.272313 \mathrm{e}^{-x} t^{3}\right) \\
u_{4}=\mathrm{e}^{-x}+1.96024 \mathrm{e}^{-x} t-0.99901\left(-0.0397532 \mathrm{e}^{-x} t-1.9602 \mathrm{e}^{-x} t^{2}\right) \\
-0.999\left(-0.000039 \mathrm{e}^{-x} t-0.041652 \mathrm{e}^{-x} t^{2}-1.3055387 \mathrm{e}^{-x} t^{3}\right)- \\
\left(-3.921 \times 10^{-8} \mathrm{e}^{-x} t-0.000080821 \mathrm{e}^{-x} t^{2}-0.0290428 \mathrm{e}^{-x} t^{3}-0.652118272 \mathrm{e}^{-x} t^{4}\right) P_{3}
\end{gathered}
$$

and so on. Fig. 2 presents the absolute errors between the exact solution $u=e^{x-2 t}$ and $7^{\text {th }}$ order OPIM approximate solution.

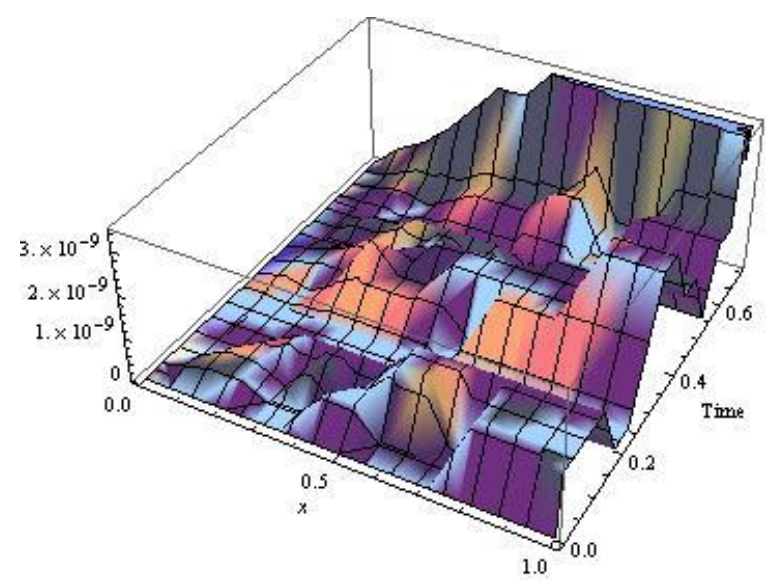

Fig. 2. Absolute errors for seventh order OPIM solution for example 2.

\section{Conclusion}


In this paper, optimal perturbation iteration method is implemented to get the approximate solutions of homogenous linear Korteweg-de Vries like equations. We analyze two examples to prove the effectiveness and applicability of the proposed technique. Figures clearly display the accuracy in higher order approximate OPIM solutions. Mathematica 9.0. is used to deal with the calculations due to huge amount of computations. We can lastly say that this work proves the flexibility and potential of the OPIM for complicated nonlinear problems in engineering.

\section{References}

[1] Bildik, N., \& Deniz, S. (2013). Applications of Taylor collocation method and Lambert W function to the systems of delay differential equations. Turkish Journal of Mathematics and Computer Sciences, 1(1), 1-13.

[2] Dehghan, M., Jalil, M., \& Abbas, S. (2010). Solving nonlinear fractional partial differential equations using the homotopy analysis method. Numerical Methods for Partial Differential Equations, 26(2), 448-479.

[3] Deniz, S., \& Bildik, N. (2014). Comparison of Adomian decomposition method and Taylor matrix method in solving different kinds of partial differential equations. International Journal of Modelling and Optimization, 4(4), 292-298,

[4] Bildik, N., \& Sinan, D. (2015). Implementation of taylor collocation and adomian decomposition method for systems of ordinary differential equations. AIP Conference Proceedings. Eds. Theodore E. Simos, and Charalambos Tsitouras (Vol. 1648. No. 1). AIP Publishing.

[5] Liao, S. (2003). Beyond Perturbation: Introduction to the Homotopy Analysis Method. CRC Press.

[6] Bildik, N., \& Deniz, S. (2015). Comparison of solutions of systems of delay differential equations using Taylor collocation method, Lambert $\mathrm{W}$ function and variational iteration method. Scientia Iranica. Transaction D, Computer Science \& Engineering, Electrical, 22(3), 1052.

[7] Bildik, N., \& Sinan, D. (2015). Modified Adomian decomposition method for solving Riccati differential equations. Review of the Air Force Academy, 3, 21.

[8] Abbasbandy, S. (2006). The application of homotopy analysis method to nonlinear equations arising in heat transfer. Physics Letters A, 360(1), 109-113.

[9] Bildik, N., \& Sinan, D. (2015). On the asymptotic stability of some particular differential equations. International Journal of Applied Physics and Mathematics, 5(4), 252.

[10] Bildik, N., \& Sinan, D. (2016). The use of Sumudu decomposition method for solving predator-prey systems. Mathematical Sciences Letters, 5(3), 285-289.

[11] Deniz, S., \& Necdet, B. (2016). Application of Adomian decomposition method for singularly perturbed fourth order boundary value problems." AIP Conference Proceedings (Vol. 1738, No. 1). AIP Publishing.

[12] Eilbeck, C. (1998). Retrieved from the website: http://www.ma.hw.ac.uk/ chris/scott_russell.html

[13] Deniz, S., \& Bildik, N. (2017). Applications of optimal perturbation iteration method for solving nonlinear differential equations. AIP Conf. Proc., 1798, 020046.

[14] Bildik, N., \& Deniz, S. (2017). A new efficient method for solving delay differential equations and a comparison with other methods. The European Physical Journal Plus. 132(51).

[15] Deniz, S. (2017). Optimal perturbation iteration method for solving nonlinear heat transfer equations. Journal of Heat Transfer-ASME, 139(37), 074503-1.

[16] Deniz, S., \& Bildik, N. (2017). A new analytical technique for solving Lane - Emden type equations arising in astrophysics. Bulletin of the Belgian Mathematical Society - Simon Stevin, 24(2), 305-320.

[17] Bildik, N., \& Deniz, S. (2017). A practical method for analytical evaluation of approximate solutions of Fisher's equations. ITM Web of Conferences, 13(01001). 
[18] Deniz, S., \& Bildik, N. (2018). Optimal perturbation iteration method for Bratu-type problems. Journal of King Saud University - Science, 30(1), 91-99.

[19] Bildik, N., \& Deniz, S. (2017). Solving the Burgers' and regularized long wave equations using the new perturbation iteration technique. Numerical Methods for Partial Differential Equations.

[20] Kaya, D. (1999). On the solution of a Korteweg-de Vries like equation by the decomposition method. International Journal of Computer Mathematics, 72(4), 531-539.

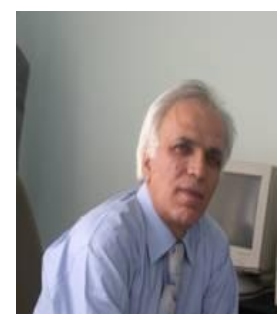

Necdet Bildik was born in Sivas/Turkey in 1951. He graduated from Ankara University in 1974. He earned the M.Sc. degree in University of Louisville, Kentucky, USA in 1978. He awarded the Ph.D. degree in Oklahoma State University, USA in 1982. He was assistant professor in 1988 and also he was became associate professor in 1995. He was promoted to be professor in 2003. He is interested in numerical analysis, ordinary, partial and non-linear differential equations, ergodic theory, stability theory.

He has over than a hundred published articles in the national and international journals and conferences. He also serves as a reviewer for many international journals. 